\title{
An Encapsulation for Reasoning, Learning, Knowledge Representation, and Reconfiguration Cognitive Radio Elements
}

\author{
Keith E. Nolan* \\ CTVR \\ Trinity College Dublin \\ keith.nolan@ctvr.ie
}

\author{
Paul Sutton \\ CTVR \\ Trinity College Dublin \\ suttonpd@tcd.ie
}

\author{
Linda E. Doyle \\ CTVR \\ Trinity College Dublin \\ ledoyle@tcd.ie
}

\begin{abstract}
State and contextual awareness, reasoning and conclusions formation, and a means of directing application, structural and parameter-level radio reconfiguration are key elements of a cognitive radio. This paper describes a cognitive radio design capable of scaling between the two extremes of minimal cognitive capabilities and complex highly-evolved cognitive radio abilities, which is being adopted for real tests using licensed cognitive radio test spectrum. A memory element stores state, sensor, objectives, actions and conclusions information and the relevance of this information can be varied in order to identify or ignore common traits or occurrences. The decisionmaking and conclusions formation abilities of this cognitive radio design can use (or choose to ignore using the variable weighting facility) external information relating to the network, and etiquettes in conjunction with the memory element. A set of actions formulated by the reasoning and conclusions formation stages direct the radio reconfiguration. This design is implemented using a General-Purpose Processor (GPP) platform as it currently offers the very high level of reconfigurability required for very malleable cognitive radio design.
\end{abstract}

\section{Introduction}

This section introduces the idea of cognition and identifies the core requirements for a cognitive radio.

Cognition in signal-processing and system control terms is the ability to develop contextual and environmental awareness aiding the development of an optimal solution for a particular problem, recognise developing patterns of

${ }^{*}$ This material is based upon work supported by Science Foundation Ireland under Grant No. 03/CE3/I405 as part of the Centre for Telecommunications Value-Chain Research (CTVR) at Trinity College Dublin, Ireland. behavior, respond to the time-varying nature of wireless channel and user activity and learn from previous experiences. Considered in isolation, the foundations of each of the core observe, orient, react and learn stages of the cognition cycle first described by Mitola [1] and more recently by Haykin [2], are not new concepts. However, it is the innovative application of a combination of these techniques in a cognitive wireless communications context that is innovative. Observation information can be derived from internal radio and system activity (including available resources, radio capabilities and spectrum activity detected at the receiver), and external sources (including external environmental sensors, policies, network-level information).

An implementation of a cognitive entity requires a highly-reconfigurable core, which can change and evolve according to the orient, react and learn stages in the cognitive cycle. Popular approaches taken in relation to how and why changes are necessary are based on game-theoretic [5], genetic algorithmic [6], Fuzzy Logic [8] and artificial neural-network principles [7]. These techniques are used to find an optimum (or near-optimum) solution to a particular wireless communications problem but require a reconfigurable radio in order to implement the desired changes and analysis the implications of this change. It is feasible that fully-engaged cognitive abilities are always required depending on the complexity of, and challenges presented by particular scenarios. Therefore, the ability to change between minimal and complex cognitive behavior can potentially reduce power consumption and increase the operating lifetime of the device.

Section 2 describes the reconfigurable core, which is the key enabling feature for this cognitive wrapper, Section 3 is a description of the core elements of the cognitive wrapper including the awareness processing, learning and action initiation stages. In Section 4 presents some conclusions from this work. 


\section{Reconfigurable Radio}

This section briefly describes the term reconfigurable radio and one instance of an actual system that is used as the basis for the cognitive wrapper described in this paper.

The term reconfigurable radio is used in this paper to describe a heteromorphic radio signal-processing chain implemented in software, connected to a minimal hardware RF front-end that may itself be reconfigurable through physical change or under software control. A reference design and implementation of a reconfigurable radio used as the basis of the reasoning wrapper design in this paper is called Implementing Radio In Software (IRIS) [9]. This system uses eXtensible Markup Language (XML) [3] to describe a radio in terms of a signal-processing chain of elements called $R a-$ dio Components. Examples of existing Radio Components include modulators, demodulators, access schemes, filters, signal conversion, source and sink elements. These Radio Components can either be sourced from a local inventory of available Components (created by the designers) or from one or more remotely-located inventories using a wired link. Each of these elements has a common architectural framework facilitating rapid development and straightforward internal creation, execution and tear-down processes.

The IRIS system caters for a hierarchy of possible reconfiguration tasks called action sets. These action sets are developed by the observations reporting, awareness processing and reasoning engine loop as depicted by Fig. 1. Application reconfiguration allows the replacement of an entire signal-chain with another desired signal chain in order to change the active application. Component reconfiguration enables one or more signal-chain processing elements (Radio Components) to be removed/replaced/added at will. This reconfiguration can during run-time in addition to the trivial static-case reconfiguration scenario. Dynamic parameter-level reconfiguration is also possible and all relevant parameters used in each Radio Component can be changed on demand. These reconfiguration possibilities allow the radio core to be molded into any form according to the instructions of a higher-level entity (cognitive wrapper), which in turn is possibly in response one or more reconfiguration triggers or drivers.

The higher-level IRIS entity governing change within the reconfigurable core is called Control Logic [9]. This is a software mechanism that implements the replace/add/remove Radio Components and controls the cascade of reconfiguration required when parameters are changed within one or more Radio Components that may impact on other Radio Components further along the signalchain. This Control Logic has been expanded to cater for a reasoning engine, memory delay-line and external input interfaces as described in this paper.

\subsection{Reconfiguration Drivers}

Preparing for the possibility of change within a cognitive radio does not imply that continuous change is required during the operating lifetime of the radio. It is conceivable that a static architecture is sufficient in some cases. Reconfiguration activity is triggered when the cognition engine determines that an observed event(s) or states necessitate an application, component or parameter change. It is necessary to drive these reconfiguration processes in an intelligent manner that will result in the implementation of a desired preset feasible solution or a solution developed by the reasoning and conclusions formation engine. Reconfiguration drivers are not limited to internal device events/observations however, and can account for radio, network, regulatory and physical environment changes, and application, business and social context changes.

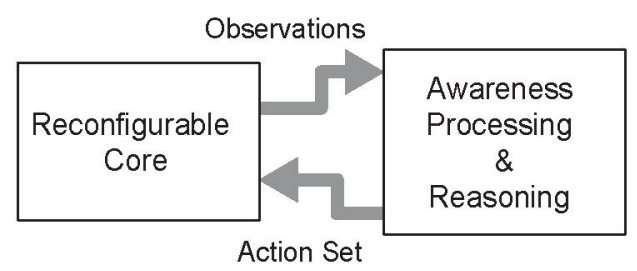

Figure 1. Reconfigurable core showing inputs (set of actions) and outputs (device state, capabilities and spectrum observations)

\section{Cognitive Wrapper}

In this section, the primary contribution of this paper is presented in more detail. This is a realisable cognitive wrapper with scalable-'intelligence' and designer-specified learning and reasoning algorithm capabilities. This section describes the core entities comprising this cognitive wrapper design, where the key fundamentals of the design are shown in Fig. 2.

The cognitive wrapper described in this paper encapsulates a reconfigurable core, which in this case is the IRIS system. Features of this wrapper, as illustrated in Fig. 2 include the observation, awareness and knowledge representation mechanisms, a variable-length memory delayline used to store current and historical knowledge sets, which can also be used to identify (or disregard) common traits/characteristics. The reasoning engine generates the reconfiguration tasks and directs these changes in the reconfigurable core. This diagram also illustrates that constraints which can include etiquettes for radio-behavior, a measure 
of the system capabilities and regulatory policies can also have a direct influence on the reasoning and conclusionsformation processes.

Reasoning tasks include developing the sequence of application, structural and parameter changes (action sets) or deciding that no reconfiguration is necessary. We consider a full-featured highly-involved cognitive radio device for the following descriptions of the reasoning wrapper capabilities.

Implementation of a cognitive system requires awareness-formation, reasoning and learning, and conclusions-development capabilities. A cognitive radio therefore requires a means of observing the environmental, social, user, spectrum and policy landscapes as described in Section 2.1, memorising (or choosing not to remember) previous events, actions and consequences, decision-making and conclusions-formation. The ability to mould the reconfigurable core by executing actions that direct the operation and structure of this core is also a high priority objective. For maximum system flexibility, the radio device should have the ability to scale the influence of the cognition capabilities between the two extremes of a highly-involved cognitive radio to a basic device with no cognition capabilities.

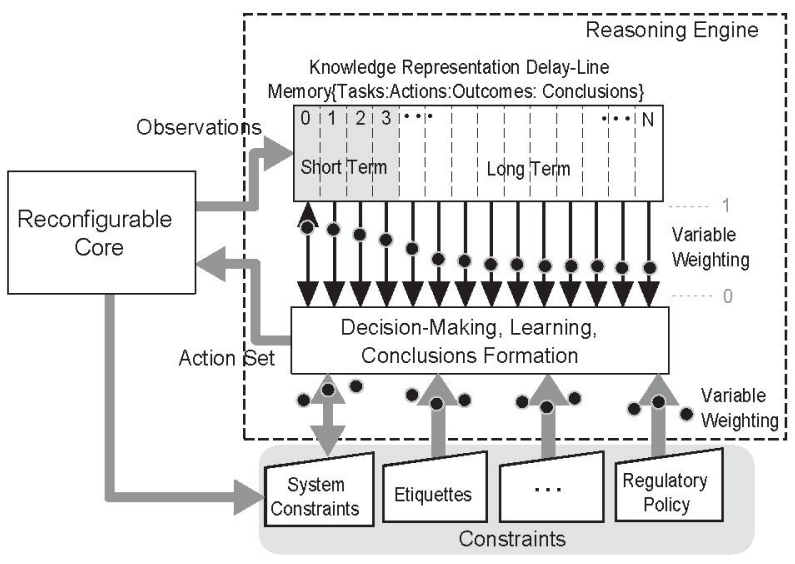

Figure 2. Reasoning wrapper overview illus-
trating the knowledge representation delay-
line, reasoning and learning engine, con-
straints, and reconfigurable core entity

A highly-evolved cognitive radio can employ contextual reasoning to help determine the best course of action to take. Interpretation of selected internal and external physical, spatial, environmental, political and objectives is therefore necessary to develop and maintain contextual awareness during the lifetime of the cognitive radio. Observations may originate from internal and external sources. The set of internal source information includes available energy, exist- ing components, data-type descriptions, available processing power, RF front-end capabilities, networking capabilities and fixed, mobile, nomadic mobility status information.

Extra sensing information can be obtained from environmental, spatial and biometric sensors including temperature, pressure, air and water quality, shock and vibration information. Spatial awareness is not limited to geographical location but include trajectories, altitude and device-tilt information. Awareness of the time-value of this information is a critical factor in the cognitive control mechanisms. Available spectrum may have a finite usage window, reaction to a sudden shock experienced by the cognitive radio may require immediate countermeasures and a device faced with a dwindling energy supply may have to initiate graceful degradation or backup measures before the remaining energy is depleted fully. Instead of the power-inefficient case where all possible sensing sources are activated at all times, the cognitive radio must be capable of focusing its resources of sensing sources deemed important at any particular time and deactivate sensing sources considered irrelevant.

\subsection{Knowledge Representation}

Aspects of the radio, including current and previous radio states, radio resources, and internal and external observation are knowledge sources used as part of the radio cognition processes. It is the relevance of this information in a particular context, instance or period of time, or scenario that influences the value of this knowledge however. An ability to store the sequence of actions taken and measurable consequences of these actions is therefore a valuable asset. Information derived from some source entity often has a strict description syntax. In order to interpret this information correctly therefore, devices must conform to a common syntactical convention. XML (eXtensible Markup Language) for example, is a portable method of representing information, which can be parsed by software processes and is presented in a human-interpretable form [3]. Web Ontology Language (OWL) is a method of representing information that does not necessarily have to be presented in a human-readable form but this information is essentially derived from an English language description of the scenario or task [4]. OWL offers a means of specifying the semantics of a scenario, which can be conveyed and translated by platforms with different syntax conventions.

The ability to store, order, extract and reuse information relating to current and historical state, actions, conclusions, objectives in a structured format facilitates application of this information in the cognitive decision-making processes. Ultimately this enables the cognitive radio to make better operational decisions. Knowledge of previous actions, and consequences of these actions also aids the 
forward-planning and anticipative action of the cognitive radio. Emerging problems can be decomposed into a set of problems with less complexity. Solutions to these nested challenges may already exist within the stored knowledge sets thus potentially reducing the overall solution-formation time.

The memory delay-line shown in Fig. 2 is the means used to store current and historical knowledge sets for the cognitive radio system described in this paper. A method used to represent short and long-term knowledge, which forms part of the input and ultimately influences the reasoning wrapper outputs and desired actions. Analogous to a finite-length filter delay-line which stores current and historical knowledge sets. Information from all stored memory sets is available for use by the cognitive engine.

The relevance of certain aspects of each knowledge set stored in the memory delay-line may not be constant. A memory-merging capability offers some interesting possibilities. For some scenarios, identification of common traits, actions or consequences of previous radio reconfiguration and observed events may be more important than spurious events or actions. Selective memory can also be used to place a greater bias on recent knowledge rather than longer-term knowledge, or vice-versa. Weighting factors, analogous to filter coefficients are used to implement memory selectiveness. The selective nature is reconfigurable by varying the weighting factors associated with the knowledge set stored in each memory delay. Equation 1 is a conceptual example of this process where $y(k)$ represents the $k^{t h}$ desired parameter value/solution, $w(n)$ is the $n^{t h}$ weighting factor assigned by the reasoning engine, $x(n)$ is the $n^{t h}$ knowledge set element stored in the memory delayline and $N_{\text {delays }}$ is the memory-length. The two extreme cases of 1 . a memory-less radio device is achievable by assigning a weighting factors of zero for all memory delayline weights and 2. a photographic memory is achievable by assigning a weighting factor of one for all memory delayline weights and averaging the result.

$$
y(k)=\sum_{n=0}^{N_{\text {delays }}} w(n) x(n)
$$

\subsection{Decision Making, Learning and Con- clusions Formation}

The main objective of the decision making, learning and conclusions formation element of the reasoning wrapper is to produce an 'intelligent' and timely answer to a problem set based on previous actions and consequences, current observations and objectives and descriptions of the data-types used for the desired wireless communications task.

This cognitive stage, illustrated in Fig. 2 is designed to adopt any feasible game-theoretic, genetic algorithmic, arti- ficial neural networks, Bayesian or Fuzzy system logic implementation approach. This is achieved using the Control Logic interface that provides the means by which, external processes can attach to, and direct the reconfigurable core. This stage can also be de-activated using this Control Logic interface if a minimal-cognition or non-cognitive device operation is required.

The feasibility of a decision making, learning and conclusions formation approach is dependent on the time required to present viable solutions and the implementation complexity associated with each approach. The presentation of a solution approaching optimality within the time constraints allowed has a potentially greater value than an optimal solution that is produced too late i.e. after the implementation deadline. Complexity and the processing burden can be reduced by implementing some features of a chosen approach. It is conceivable that significant gains using dynamic spectrum access techniques can be achieved without the full weight of a maximal-complexity cognitive engine. The platform presented in this paper offers the ability to investigate the real achievable spectral-efficiency gains using actual RF spectrum in a controlled interference and user-activity environment. The cost function determining the real increase in spectrum-usage efficiency can be reconfigured permitting the exploration of many different case studies. Examples of these include investigating the cost of rapid spectrum allocation where processing power is the determining factor and investigating the cost of opportunistic access of narrow spectrum segments where interference may be the important factor.

A cognitive radio faced with a developing wireless communications scenario may be forced to expend considerable energy using resources on determining the best course of action. A better approach is to break down the developing situation and apply a sequence of less complex incremental solutions. The objective in this case is to solve the complex overall problem using a combination of these incremental solutions. The possibly complex scenario can decomposed into two main classes, where incremental solutions for each stage may already exist in the delay-line of knowledge sets. The first case is a repeatable scenario and the second case is a unique scenario.

A repeatable scenario is where similar wireless communications tasks and observable environmental conditions occur more than once. In this case, the cognitive radio, which identifies the emerging similarities from the knowledge sets, can invoke a sequence of previously successful procedures in an attempt to accelerate the completion of communications task(s). The potential benefits of this ability include conservation of radio resources, increased usersatisfaction levels and possibly reduced interference levels to other wireless devices as the signalling-overheads may be reduced as a consequence. 
Table 1. Knowledge Set Example

\begin{tabular}{c||c||c||c}
\hline \multicolumn{1}{c||}{ Observations } & Tasks & Actions & Policies \\
\hline \hline BW: 2MHz, Freq: 2.08GHz & Voice: High quality & OFDM: 50\% user share & Interference Avoidance \\
\hline Avail. energy:high & Reconfigure: OFDM & TX Power: min. & Social: extrovert \\
\hline Users: 2 & Maintain link & Sense before use & - \\
\hline Mobility: mobile & - & - & - \\
\hline
\end{tabular}

The unique scenario is where internal and external observations, available radio resources and desired communications task are not the same (or in the same sequence) as previously experienced by the cognitive radio. It is possible that the unique scenario may be decomposed as a sequence of repeatable scenarios. Thus the cognition wrapper can attempt a combination of proven tactics in order to provide a solution.

The knowledge gained from a communications task may include the data-type involved, power and duration of the transmission, structural, component and parameter configuration of the radio that successfully (or unsuccessfully) completed the task. The consequences of this communications task can include Bit Error Rates (BER), Quality of Service (QoS), consumed radio resources, interference experienced or inflicted, and an estimate of the spectrum efficiency.

An action set is the list of required components, structural configurations or possible reconfiguration instructions for existing structures, component parameter-values and deadlines by which the action set should be implemented. Action sets are produced by the reasoning and learning mechanisms employed in the cognitive engine. The heteromorphic reconfigurable radio core is instructed to execute an action set using Control Logic. Control Logic is the means of addressing each structure, component and parameter capable of being implemented by the reconfigurable core. The action set for an OFDM receiver can include the entire set of required components, or incremental changes to an existing structure, required parameter values, and the time-span in which to implement the action set.

\section{Conclusions}

This paper has presented a cognitive wrapper encapsulating a highly reconfigurable radio core. The influence of this wrapper can be varied in order to vary the 'intelligence' of the cognitive radio between the two extremes of a baseline radio, and a highly-complex and evolved system to account for scenarios where complex cognition may not be necessary. Observations, actions and conclusions developed by the cognitive radio can be stored in a variable-length memory delay-line. A selective memory mechanism enables the relevance of aspects of this information to be varied.

\section{References}

[1] Mitola, J., III; Maguire, G.Q., Jr., "Cognitive radio: making software radios more personal," Personal Communications, IEEE [see also IEEE Wireless Communications], vol.6, no.4pp.13-18, Aug 1999

[2] Haykin S., Cognitive Radio: Brain-Empoweredd Wireless Communications, IEEE Journal on Selected Areas in Communications, February, 2005.

[3] http://www.w3.org/XML/

[4] http://www.w3.org/TR/owl-features/

[5] J. Neel, J. Reed, R. Gilles. The Role of Game Theory in the Analysis of Software Radio Networks, SDR Forum Technical Conference November, 2002.

[6] Rieser, C.J.; Rondeau, T.W.; Bostian, C.W.; Gallagher, T.M., "Cognitive radio testbed: further details and testing of a distributed genetic algorithm based cognitive engine for programmable radios," Military Communications Conference, 2004. MILCOM 2004. IEEE, vol.3, no.pp. 1437-1443 Vol. 3, 31 Oct.-3 Nov. 2004

[7] Hopfield, J.J., "Artificial neural networks," Circuits and Devices Magazine, IEEE, vol.4, no.5pp.3-10, Sep 1988

[8] Klir, G.J., "Fuzzy logic," Potentials, IEEE, vol.14, no.4pp. 10-15, Oct/Nov 1995

[9] P. Mackenzie, "Reconfigurable Software Radio Systems", Ph.D dissertation, Trinity College Dublin, Ireland, 2004.

[10] Nolan, K.E., Reconfigurable OFDM Systems, Ph.D thesis, University of Dublin, Trinity College, May 2005. 